\title{
Computerized Tomography with Digital Lines and Linear Programming
}

\author{
Fabien Feschet and Yan Gérard \\ LLAIC - IUT Clermont-Ferrand, \\ Campus des Cézeaux \\ 63172 Aubière Cedex - France \\ ffeschet, gerard\}@llaic.u-clermont1.fr
}

\begin{abstract}
We present a new method of computerized tomography based on linear programming. The approach is based on three main ideas: covering the set of pixels by digital lines, introducing a variable of maximal error in the linear constraints and adding in the objective function an entropy term.
\end{abstract}

\section{Introduction}

Since the invention of the first X-rays scanner by G.N. Hounsfield and A. McCormack in the beginning of the seventies, computerized tomography has become an industrial, medical and mathematical stake. The principle of this technology is to reconstruct an image from X-rays. This purpose requires to solve a mathematical problem belonging to the family of inverse problems: compute the inverse of the radon transform. A solution is given by the formula of the Fourier slice theorem [1] but this result belongs to the framework of "continuous" mathematics whereas the technology of the measurements of the X-rays provides only discrete data. The computation of the image with the Fourier slice theorem requires to know the $\mathrm{X}$-rays for all the angles going from 0 to 360 degrees, which is of course not possible. The camera can only provide X-rays according to a finite panel of directions. This reason and the fact that the captors of the camera provide digital data with a finite precision make rational to consider the problem in a discrete framework (Fig 1).

The most popular methods of computerized tomography are the filtered backprojection algorithm and its variant called the convolution back-projection algorithm. Since the seventies, the back-projections methods have been chosen in computerized tomography in preference to iterative $[2,3,4,5]$ reconstruction techniques. These algebraic approaches $[6]$ have however recently come to the lead in both frameworks of PET imagery and discrete tomography. Discrete tomography deals with the reconstruction of binary images, namely lattice sets instead of grey-level images (see [7] for details). One of the characteristics of discrete tomography is the small number of directions which makes the Fourier analytical tools inappropriate. In this particular framework, the algebraic approach and especially linear programming has provided a lot of interesting results ([8], 

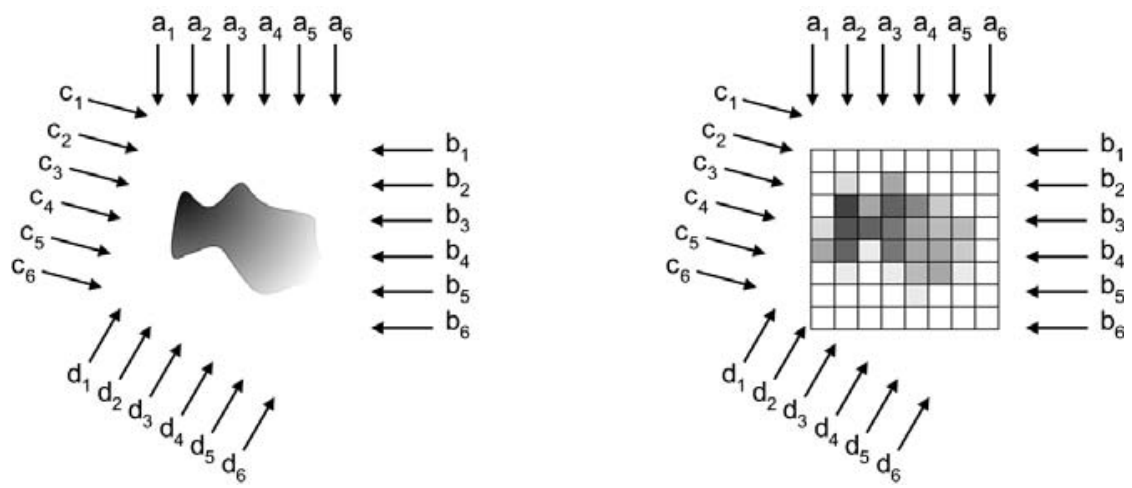

Fig. 1. Moving the camera in four positions with a panel of directions of angles 0, 90, 150 and 210 around the object provides four vectors $\left(a_{i}\right)_{1 \leq i \leq I},\left(b_{i}\right)_{1 \leq i \leq I},\left(c_{i}\right)_{1 \leq i \leq I}$ and $\left(d_{i}\right)_{1 \leq i \leq I}$ called X-rays. The purpose of tomography is to obtain from the X-rays a significative grey-level image of the object

[9], [10], [11], [12]). This paper is deeply inspired by these earlier works. Its purpose is a presentation of a new method of computerized tomography based on linear programming. The choice of reconstructing grey levels images instead of lattice sets avoids the main difficulty met by this kind of methods in discrete tomography. Then we can focus on the generation of the linear constraints and of the objective function. They are based in the new method on three elementary ideas:

- We cover the set of the pixels by digital lines (a digital line is a subset of $\mathbb{Z}^{2}$ characterized by a double-inequality $\left.\mu \leq a x+b y<\mu+\delta[13]\right)$ instead of using Diophantine straight lines or weights equal to the lengths of the intersection between lines and pixels.

- We introduce in the problem a variable of maximal error that we denote $h$. The linear constraints of the linear programming instance come from the definition of $h$ : each difference between the given X-rays and the X-rays of the image should belong to the interval between the maximal error and its opposite. The choice of the objective function is made in order to minimize $h$.

- We add in the objective function an entropy factor as done in [10].

Next section 2 is focused on the literature of linear programming and tomography. The purpose of the third section 3 is a detailed description of our new method while section 4 provides experimental results.

\section{Tomography and Linear Programming}

According to [8], the idea to use linear programming in the framework of discrete tomography came to P. Fishburn, P. Schwander, L. Shepp, and R. Vanderbei 
after a talk of A. Kuba at the first mini-symposium on discrete tomography (DIMACS, 1994, Rutgers University) about his joint work with G. Herman and R. Aharoni. It was the first intrusion of linear programming theory in discrete tomography and probably in the whole domain of tomography. Since ten years, this idea has get on well by providing a lot of interesting results going from algorithms to more theoretical questions as for instance uniqueness [8] or stability of the solutions [11]. The basic principle is to relax the binary constraint on the image for computing with linear programming a grey-level image. The instance is made from the constraint on the grey level to belong to an interval while some partial sums according to lines are equal with the given X-rays [8]. This first formulation searches only exact solutions with the disadvantage that approximations are not supported (if only one data is modified, the feasibility of the instance is not guaranteed). It makes this approach inappropriate to real data. Then P. Gritzmann, S. de Vries, and M. Wiegelmann have relaxed the equalities between the partial sums and the X-rays: whether they consider that the sums are less than the X-rays and they maximize the sum of the grey levels (method Best-Inner-Fit), or they consider that the sums are greater than the X-rays and they minimize the sum of the grey levels (method Best-Outer-Fit) [9]. This approach can deal with real data because any instance is feasible. Next point was the introduction by S. Weber, C. Schnörr, and J. Hornegger of an entropy term in the objective function. This technic requires auxiliary variables with an expensive increase in the number of constraints. The computation is much more expensive in time but it provides results that the human eyes should find more obvious and less noisy than the ones obtained without entropy. It is the reason why we kept this term for reconstructing grey levels images.

Finally, we mention other works trying to use sophisticated tools of convex optimization [5] or Difference Convex programming [14].

\section{New Method}

\subsection{Digital Lines}

To project a continuous image, real lines are used and the projected value is the integral of density along the line of projection. When dealing with discrete images, projections may be modeled with different models. First, it is possible to consider the intersection between the digital grid and rational lines $[12,9]$. In this case, a line is a set of integer points nearly always disconnected. The value of the projection is the sum of the values of the points of the intersection. Second, it is possible to consider real lines and to discretize the intersection of the lines with the discrete grid. More precisely, each point of the discrete grid might be modeled as a unit square. Then the intersection of a line with each square is an interval whose length is used as a weight associated to the integer points situated at the center of the square. This is done for instance in [14].

None of the above methods are satisfactory because discrete projections are computed with disconnected sets in the first case, and weights introduce a mix 
between the discrete space $\mathbb{Z}^{n}$ and the real space $\mathbb{R}^{n}$. We propose another strategy which is a purely discrete approach. Our projection operator is based on arithmetical lines.

The $\mu$ parameter controls the translation of any given arithmetical line over the grid whose size is the size of the image to be reconstructed. If the width parameter $\delta$ is greater than $\max (|a|,|b|)$ then the arithmetical lines are connected and hence projections are computed from connected subsets of the image. In the experiments, we have chosen to use digital lines with $\delta=\max (|a|,|b|)$ (these digital lines are called "naive"). The translation parameter $\mu$ has also a deep impact on the projection operator. Indeed, it must be increased or decreased by $\delta$ to ensure that consecutive lines do not intersect. Hence smaller values force the consecutive lines to overlap, which makes the projection operator closer to the real process of acquisition used in computerized tomography. As a byproduct, it is possible to deal with image having an imported resolution while keeping limited resolutions for the projections. This is also achieved thanks to the parameters $\delta$ and $\mu$ of arithmetical lines.

\subsection{Linear Programming}

As previously described in the paper, discrete tomography can be algebraically reduced to a problem of the form

$$
A x=b
$$

However, the matrix $A$ is rarely invertible. Hence, the previous system cannot be solved algebraically. To build an approximate solution, we introduce the error term: $e=A x-b$. Ideally $e=0$ but since this never happens, we try to minimize $e$. Minimization corresponds in fact to the minimization of the norm $\|e\|$ of $e$ for some norm $\|$.$\| which can be the L_{1}, L_{2}$ or any $L_{p}$ norm or the $L_{\infty}$ norm. $L_{2}$ norm leads to a mean-square approximation of the solution whereas the $L_{\infty}$ norm controls only the worse term of the approximation. Hence, we bound $e$ by $h \mathbb{1}$ and $-h \mathbb{1}$, where $\mathbb{1}$ is the constant vector with all components equal to one, and we minimize $h$. It is known [15] that $L_{\infty}$ norm problem might be solved by linear programming. More precisely, to minimize the $L_{\infty}$ error, it is sufficient to solve the following problem

$$
\left\{\begin{array}{l}
\min h \\
\text { such that } \\
-h \mathbb{1} \leq A x-b \leq h \mathbb{1}
\end{array}\right.
$$

This linear programming problem can be solved by any available linear programming solver. We have chosen the program soplex [16].

Approximation of the computerized tomography reconstruction problem with linear programming is an interesting approach but lacks of topological constraints [10]. 


\subsection{Entropic Regularization}

To solve this problem, regularization constraints must be added to the linear programming approach. In [10], the following strategy was used. Some variables $z_{(j, k)}$ were added for each position $(j, k)$ where $j$ and $k$ are 4-neighbors in the grid containing the image. Then, $z_{(j, k)}$ is forced to be lower than the absolute value of the difference between the values at position $j$ and $k$. Moreover, each density value is searched in the continuous interval $[0,1]$.

We have adopted another strategy to test the influence of an entropic term onto the reconstruction. First if we want to reconstruct images with $g$ grey levels, we have forced all variables in the continuous interval $[0, g-1]$. Second, let us consider a position $(m, n)$ in the grid. $x_{(m, n)}$ denotes the unknown density value at position $(m, n)$. We introduce two variables, $u_{(m, n)}$ and $v_{(m, n)}$ and we add the constraints : $u_{(m, n)} \leq \min \left(x_{(m, n)}, x_{(m+1, n)}\right)$ and $v_{(m, n)} \leq \min \left(x_{(m, n)}, x_{(m, n+1)}\right)$. Obviously, our entropic term is not symmetric whereas the previous one of Weber et al [10] was. This might result in privileging high values.

The final problem to solve becomes:

$$
\begin{cases}\min h-K\left(\sum_{(m, n)} u_{(m, n)}+v_{(m, n)}\right) \\ \text { such that } \\ -h \mathbb{1} \leq A x-b \leq h \mathbb{1} \\ u_{(m, n)} \leq \min \left(x_{(m, n)}, x_{(m+1, n)}\right), \quad \forall(m, n) \\ v_{(m, n)} \leq \min \left(x_{(m, n)}, x_{(m, n+1)}\right), & \forall(m, n) \\ 0 \leq x \leq(g-1) \mathbb{1} & \end{cases}
$$

$K$ is a constant used to control the influence of the topological constraints versus the minimization of $h$.

\section{Experiments}

We present in this section some experiments to illustrate the quality of the reconstruction method. Despite the fact that the method was designed to deal with grey level images, we have applied it successfully to black and white image usually encountered in classical discrete tomography. The method has been tested on two types of simulated images. One image was used to test the influence of the constant $K$ parameter and the other image, closed to real images acquired in practice, was used to study the influence of the number of directions in the reconstruction process.

For each test, the method was the following. Starting with the resolution of the reconstructed image, we have generated the list of constraints using non 
overlapping naive lines. Projections were computed free of error using the synthetic images. The linear programming problem was solved by soplex without any special parameters. The output of soplex was converted to an image by rounding, since the variables were forced to be in the interval of allowed grey values.

\subsection{B and W Tests}

We start with a black and white test (see Fig. 2). $K$ was fixed to 0.001. No difference was constated between the two images. When considering real values, the output of soplex was either 0 or 255 , so no rounding was in fact added to the process. It must be noticed that the reconstruction uses 8 directions: $(1,0)$, $(0,1),(1,1),(1,-1),(1,2),(2,1),(1,-2)$ and $(2,-1)$.

A second test (see Fig. 3) was done on an imperfect black and white image. We have used the same $K$ value and the same projections than for the previous experiments. Due to noise, some grey levels appears inside the objects which is in accordance to what was expected since noise perturbates the projections.

We now illustrate the influence of the constant $K$ on the reconstruction process. The image in Fig. 3 was reconstructed with $K=0.001$.In Fig. 4 , we present

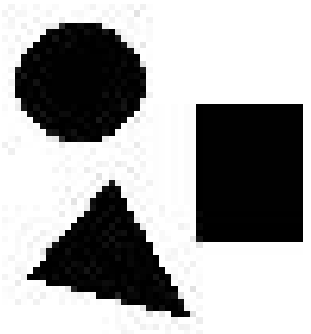

Fig. 2. (left) Original Image (right) Reconstructed Image

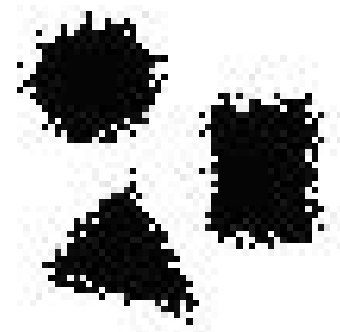

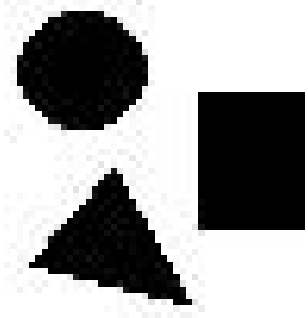

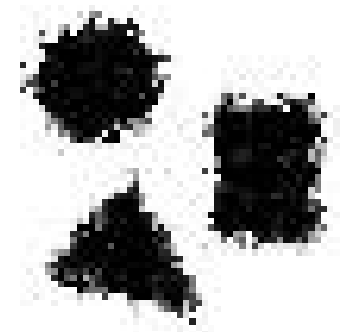

Fig. 3. (left) Original Noisy Image (right) Reconstructed Noisy Image 

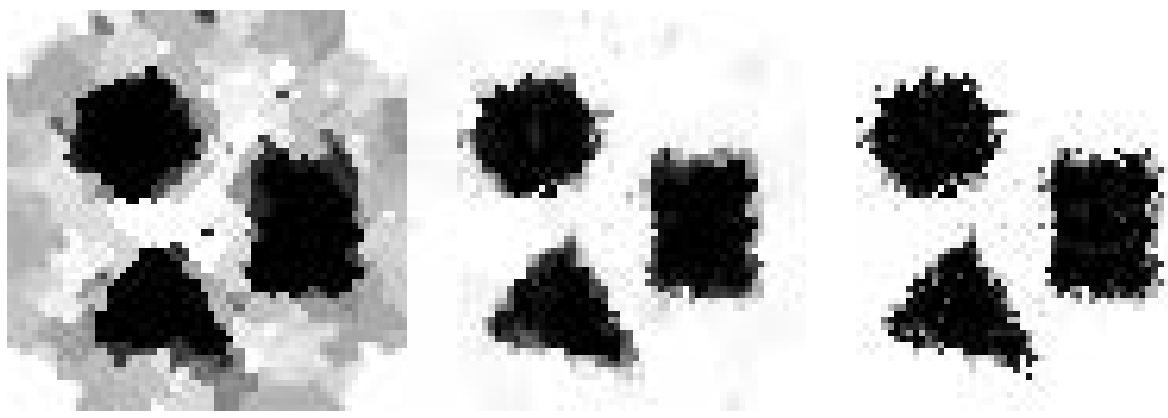

Fig. 4. (left) $K=0.01$ (middle) $K=0.007$ (right) $K=0.0001$

the reconstruction obtained for $K=0.01, K=0.007$ and $K=0.0001$. When $K$ has a too high value, the topological constraints are stronger than the constraint on $h$. Hence, this forces to have regions with regular grey level even if objects inside the image are not well reconstructed. When $K$ decreases, the regularity of the grey levels appears inside the objects but when $K$ is very small, the influence of the second term becomes neglectable and thus peaks of grey level appear. All reconstructions were done with the given eight directions.

\subsection{Grey Level Tests}

We have also tested the method on a synthetic image which has simply been constructed by adding grey levels inside the objects of the black and white noise free image. We still used 8 directions and $K$ was fixed to 0.001 . Results are given in Fig. 5. Images look similar even if they are not. The only difference is in the grey level regularity. The effect of the topological constraints was to regularize variations of grey levels in the reconstructed image. Visually, this effect was not detected first. When zooming both images (see Fig. 6), the effect of the
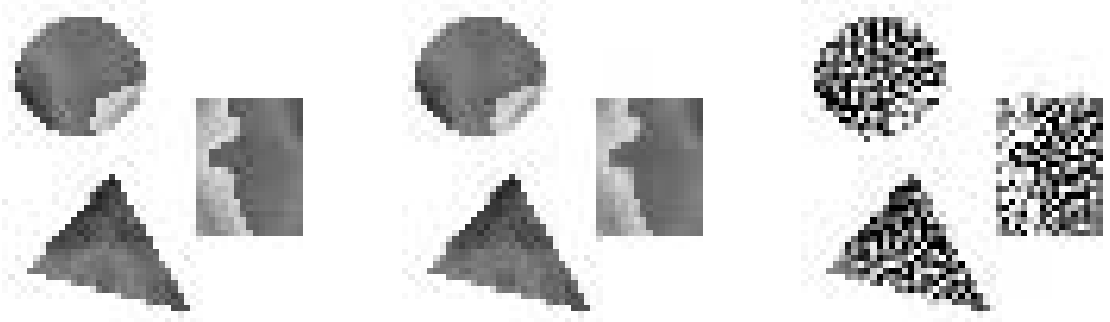

Fig. 5. (left) Synthetic grey level image (middle) Reconstruction with $K=0.001$ (right) Reconstruction with $K=0.0$ 

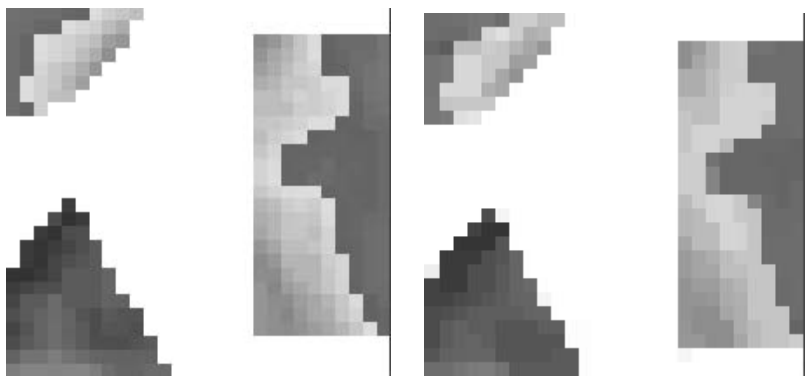

Fig. 6. (left) Original image (right) Reconstructed image

topological constraints becomes clearer in the variations of grey levels in the reconstructed images.

In Fig. 5, we provide the result obtained when $K$ is null. Hence, the linear program only corresponds to the minimization of $h$, that is the $L_{\infty}$ norm. As it can be seen, the solution is not acceptable since the reconstructed grey levels have no meaning at all. Minimizing only the error on the projections does not permit to reconstruct the grey levels inside the image.

\subsection{Influence of the Number of Directions}

We now carry on our analysis with a more realistic synthetic image by the analysis of the influence of the number of directions on the quality of reconstruction, see Fig. 7. We have used at most 16 directions by adding the following directions to the previous ones: $(1,4),(4,1),(2,3),(3,2),(1,-4),(4,-1),(2,-3)$ and $(3,-2)$. The experiments were performed by taking the first four values of the 8 directions given earlier in the paper, then by taking all previous 8 directions and finally the sixteen directions.

As it can be seen, the influence of the directions is certainly not neglectable. $K$ was fixed to a constant value $K=0.001$ and this explains the regularity of the grey levels. With four directions, it was nearly impossible to reconstruct a good image. However, a careful analysis of the images shows that the result is not so bad since high and low values of grey levels correspond inside the object of the original position of extremal values. It is clear however, that the information given by the four projections were insufficient. With eight direction, the result is better. It is also better localized. This image can be sufficient in practice but is not perfect. The area which is totally missing is the hole between the two objects. Eight projections is insufficient to separate the objects where it was sufficient to reconstruct nearly well the interior grey values of the objects. When using sixteen directions, separation of the objects appears as well as a perfect localization of grey level extrema. This result is totally coherent with what was expected. More projections can be added but it is clear that visually sixteen are sufficient. Since reducing the number of directions is a challenging problem, it seems that our method does succeed well in this task. 


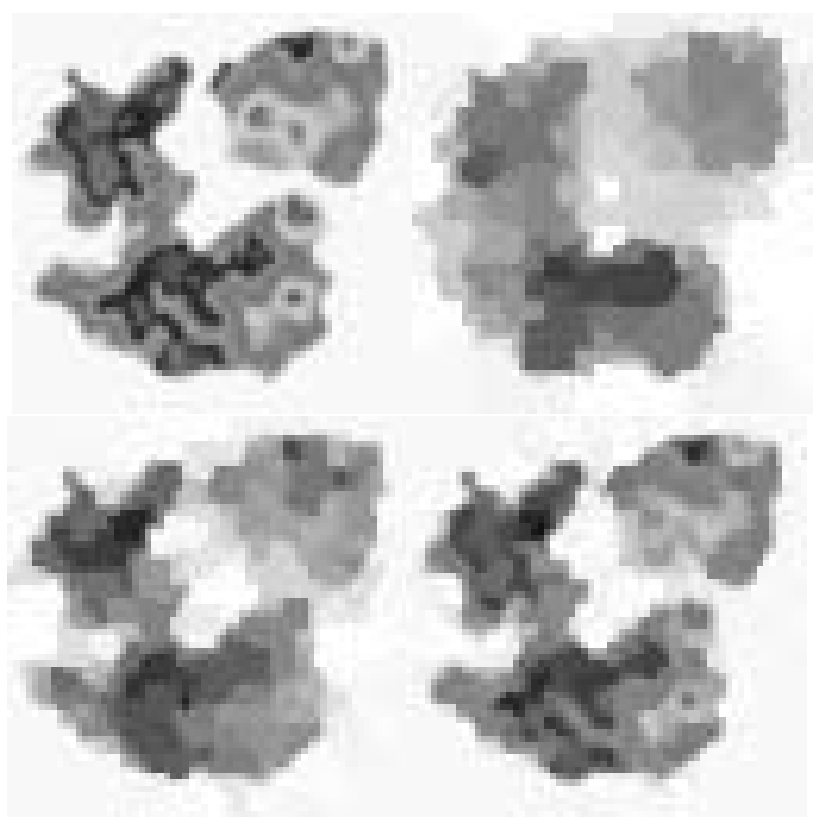

Fig. 7. In order, original image, reconstructed with 4, 8 and 16 directions

\section{Conclusion}

We have presented in this paper a promising method for reconstructing grey level images in computerized tomography. Based on linear programming and digital lines, this method has a pure discrete nature. It was successfully tested on synthetic images and seems also to perform well on black and white images. We believe that this method can be a good starting point for constructing efficient and reliable reconstruction method. All experiments were done on $64 \times 64$ images but is virtually not limited. Times of computations approach one hour with soplex at this time but the solution is nearly obtained in 20 minutes. Computing time compatible with clinical available time is a challenging problem that must be addressed in future works.

\section{References}

1. Kak, A., Slaney, M.: Principles of Computerized Tomographic Imaging. IEEE Press (1988)

2. Herman, G., Lent, A.: Iterative reconstruction algorithms. Comput. Biol. Med. 6 (1976) 273-274

3. Gordon, R., Herman, G.: Reconstruction of pictures from their projections. Communication of the ACM 14 (1971) 759-768

4. Gordon, R.: A tutorial on ART (Algebraic Reconstruction Techniques). IEEE Transactions on Nuclear Science NS-21 (1974) 31-43 
5. Ben-Tal, A., Margalit, T., Nemirovski, A.: The ordered subsets mirror descent optimization method with applications to tomography. SIAM J. Optimization 12 (2001) 79-108

6. Aharoni, R., Herman, G., Kuba, A.: Binary vectors partially determined by linear equation systems. Discrete Mathematics 171 (1997) 1-16

7. Kuba, A., Herman, G., eds.: Discrete Tomography: Foundations, Algorithms and Applications. Birkhaüser (1999)

8. Fishburn, P., Schwander, P., Shepp, L., Vanderbei, R.: The discrete radon transform and its approximate inversion via linear programming. Discrete Applied Math. 75 (1997) 39-61

9. Gritzmann, P., de Vries, S., Wiegelmann, M.: Approximating binary images from discrete X-rays. SIAM J. Optimization 11 (2000) 522-546

10. Weber, S., Schnörr, C., Hornegger, J.: A linear programming relaxation for binary tomography with smoothness priors. In: Int. Workshop on Combinatorial Image Analysis IWCIA'03. Volume 12 of Electronic Notes in Discrete Math., Elsevier (2003)

11. Brunetti, S., Daurat, A.: Stability in discrete tomography: Linear programming, additivity and convexity. In: 11th Discrete Geometry and Computer Imagery. Volume 2886 of LNCS., Springer-Verlag (2003) 398-408

12. Hajdu, L., Tijdeman, R.: An algorithm for discrete tomography. Linear Algebra and Appl. 339 (2001) 147-169

13. Reveillès, J.P.: Géométrie discrète, calcul en nombres entiers et algorithmique. Thèse d'état, Université ULP - Strasbourg (1991)

14. Weber, S., T. Schüle, C.S., Hornegger, J.: Discrete tomography by convex-concave regularization and d.c. programming. Technical report, Mannheim University (2003)

15. Schrijver, A.: Theory of Linear and Integer Programming. J. Wiley and Sons (1986)

16. Wunderling, R.: Paralleler und Objektorientierter Simplex-Algorithmus. PhD thesis, ZIB TR 96-09, Berlin (1996) 